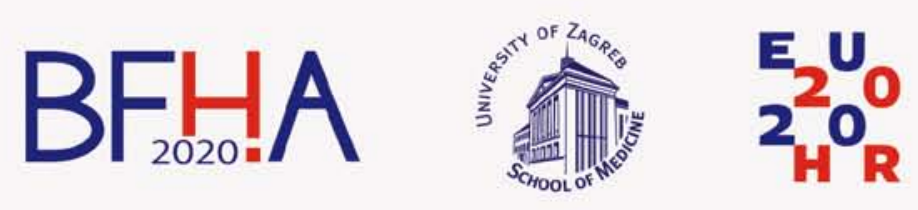

BETTER FUTURE of HEALTHY AGEING 2020

Theme B

Smart Technologies for Age Friendly Ecosystems

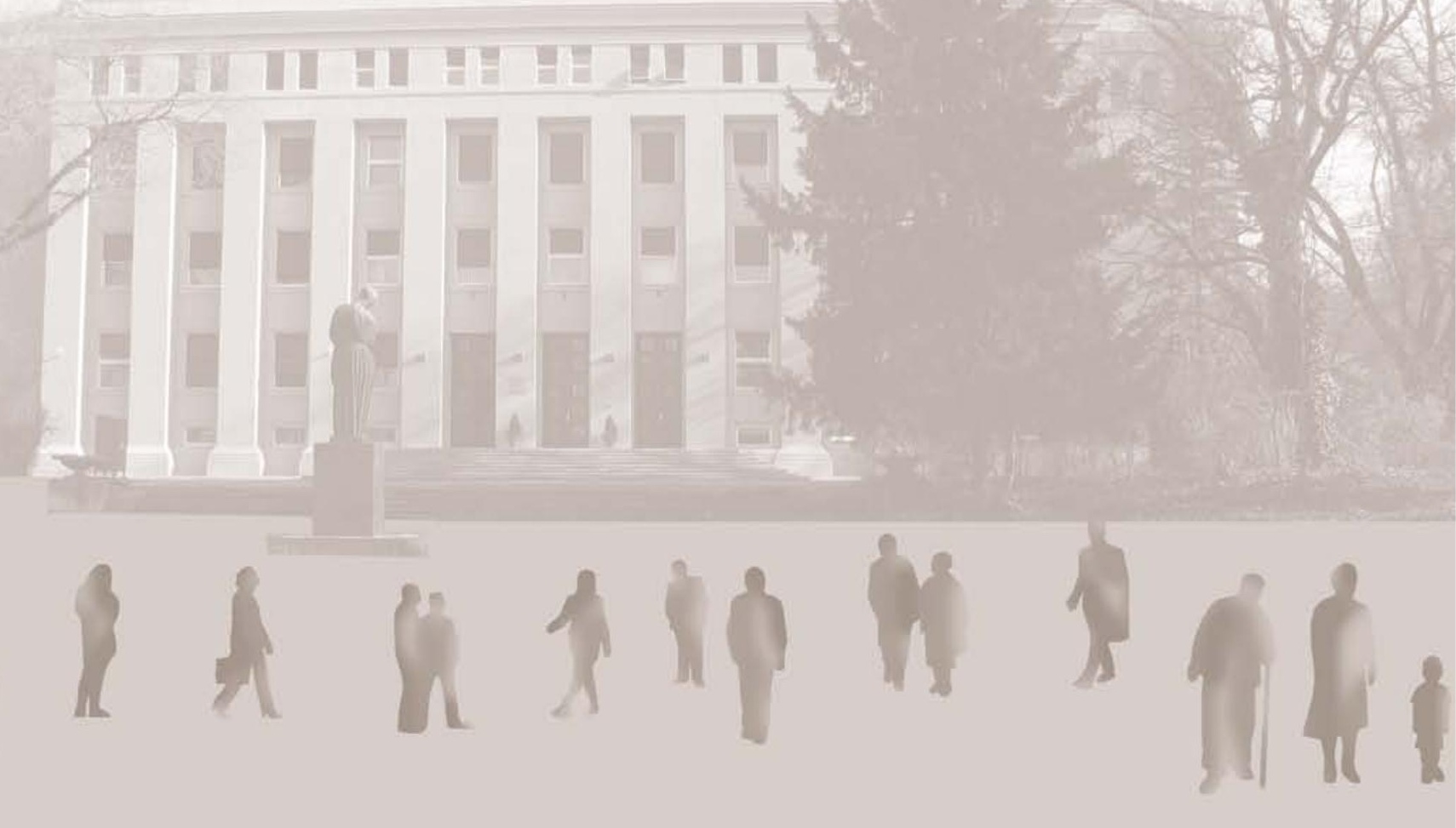





\section{Invited lecture}

\section{B1-I \\ IT Technologies in the Service of Healthy Ageing}

\section{Giuseppe Fico \\ Life Supporting Technologies, Spain}

Throughout Europe and all around the world, mortality rates have fallen significantly over the past decades [1] leading to considerable changes in the age distribution of societies [2]. If these added years are dominated by rapid declines in physical and mental capacity without support, the implications for older people and for society are further more negative. Responding to the burden of ageing presents challenges for all health systems. More emphasis on the prevention of diseases and frailty states, on new ways to reorient the provision of care, is needed. Strategies that involve multiple organizational aspects, that are setting shared decision making processes in the community, and incorporate a multidisciplinary team approach are more likely to be effective[3]. Among the few models that have proved worldwide to be effective in implementing paradigm shift, the Chronic Care Model $(\mathrm{CCM})$, and its adaptations and variations, is the mostly used by several regions to reform health care system with a long-term and 'big' plan, to use the full potential of a single-payer system [3]. The CCM is one of the few models that conceptualized effective components of care, even though these components have been not structured, and have not been well and formally articulated. "Consequently, there is limited evidence about the real impact of any of the existing models" [4]. Is it possible to improve the way evidencebased healthcare impacts our systems? In this context, innovations could support to achieve better diagnosis, treatment and management for patients across the continuum of care. Despite the exponential and astonishing advances in medical technologies 1 , the way they are delivered is not sufficiently efficient, effective and consumer friendly. In a famous Harvard Business Review editorial, three types of innovations (technological, services and business) and six forces (players, funding, technology, customers, accountability) should be analysed in order to understand how to introduce innovations to improve $\mathrm{HC}$ systems, mentioning that In Europe there are serious barriers towards adoption of customer-based innovations, technology-based are restricted and regulated by Health Technology Assessment agencies, due to cost expenditure maintenance, causing the absence of a large venture capital community; however, the centralized nature of European HC systems offers the potential for innovation in services for long term treatment and chronic diseases [5]. Nevertheless, technological innovations have proved to be the major contributor to the growth of countries in the last two centuries, especially when they have been part of common evolutionary trajectories and when they are grouped into paradigms [6], meaning that they are identified as "activities to solve problems that are selected by the paradigm itself" [7], acting as catalyst for innovations. Why this is not happening enough in the healthcare sector? The improvement of innovation delivery involves the creation of multidisciplinary research teams and taskforces, rather than just working teams [8]: multidisciplinary research involves "not only the integration of discipline-specific approaches, but also the extension of these to generate fundamentally new conceptual frameworks, hypotheses, theories, models, and methodological approaches that transcend their disciplinary origins" [9] and new challenges in evaluating the processes, outcomes, and skill acquisition of members of multi-disciplinary teams" [10]. Over the past decade, data scientists and engineers have become increasingly drawn to and involved with healthcare. This interest has recently been accelerated by the now near universal digitization of healthcare, providing data scientists with a toehold and a progressively important role in daily care as well as research. In the same period, the European 1 Stanford Medicine 2017 Health Trends Report. http://med. stanford.edu/school/leadership/dean/healthtrends. $\mathrm{html}$ Commission has been organizing a series of multidisciplinary activities to drive the adoption of innovations towards improvement of healthcare systems and outcomes, such as the European Innovation Partnership on Active and Healthy Ageing (EIPonAHA), the European Institute of Technology for Health (EITHealth), the eHealth Action Plan 2012-2020, the Smart Living Environment for Ageing Well working group of the Alliance for Internet Of Things Initiative (AIOTI), the Study on Big Data in Public Health, Telemedicine and Healthcare. Despite all these huge efforts, no one is trying to "connect all the dots", and there is the risk that each one of these initiatives claims 
to be holistic and breaking silos in a syncretistic manner. How can this group of diverse talents, interests, and schedules be brought together for productive collaboration? The digitization of healthcare provides data scientists with a toehold and a progressively important role in daily care as well as research. How can this group of diverse disciplines, sectors, talents, interests, and schedules be brought together for productive collaboration? The main objective of this speech is to show how effective implementation of healthcare interventions can be driven through structured adoption of innovation and technological paradigms, unbinding the potential of the synergies between these domains.

Four main research activities are proposed: 1. Translate Evidence-Based Healthcare (EBH) into actionable knowledge through eHealth paradigm wide and successful adoption of effective models of care is only possible when research findings can be translated. In order to transform knowledge and evidence into action, the way EBH is valued, synthesized, transferred and delivered is crucial. This means identifying a conceptualized model of care, and a suitable knowledge translation process, from discovery to intervention development, from development to delivery. The eHealth paradigm is currently used by the EC to improve efficiency of healthcare systems 2 . 2. Face the complexity of healthcare through multidisciplinary research and teams, empowered by the Internet of Things and Big Data paradigms: once concepts are defined, it is then possible to understand which domains and disciplines are (or can be) involved, in order to better analyse the way effective interventions can be put in place. The Big Data and Internet of Things paradigms, sometimes seen as two sides of the same coin, would allow multidisciplinary teams to use data when it is still in motion, and extract valuable information from it, as a sort of knowledge in motion. 3. Turn knowledge and actions into Decision Support Systems, making use of Artificial Intelligence: once concepts, roles and knowledge are defined, it is possible to create intelligence systems through the AI paradigm. Rule inference and machine learning techniques can be used to create common and well-defined intelligent systems that could accelerate the innovation processes and provide recommendations to stakeholders and end-users. 4. Validate the DSS retrospectively and prospectively, and define new ways of assessing healthcare innovations throughout their whole life cycle. Once the DSS is developed, verifying how can support stakeholders and end-users of healthcare systems in taking better decisions and with which results, are fundamental activities. Understanding this means to define new ways of evaluating innovations in all aspects of healthcare (technologies, services, organizations).

\section{R E F E R E N C E S}

1. European Commission: The 2015 ageing report, underlying assumptions and projection, methodologies. (2014).

2. United Nations. "World population ageing: 1950-2050." New York: Department of Economic and Social Affairs (2002).

3. Rechel, Bernd, et al. "Ageing in the European union." The Lancet 381.9874 (2013): 1312-1322. 


\section{Oral presentations}

\section{B1-01 \\ Not the Computer but the Place: Designing and Evaluating Future Ambient Spaces for Improved Quality of Life in the Elderly}

\section{John Waterworth \\ Department of Informatics, Umeå University, Umeå, Sweden}

Introduction and objectives. In a set of related studies, we designed, implemented and evaluated ambient environments that support the wellbeing of elderly citizens: a) Exercise and health. Engaging in regular physical exercise improves not only physical wellbeing but also mental health problems such as depression and is a preventative factor in cognitive deterioration. b) Social engagement. Loneliness and social isolation are risk factors for elderly wellbeing. c) Experienced safety and privacy when using networked technologies. Methods. a) We designed and evaluated medically-recommended exercise programs based on sensing technologies in the user's home or in public spaces. In one study, outcomes in Spain and Sweden were compared. In another, exercise games with an added cognitive task were compared with similar games without. b) We designed and evaluated a range of interactive tools in the homes of users: a custom social network, ambient devices for signalling the arrival of messages or the need to communicate, and camera-based mood recognition. c) We are exploring attitudes to internet threats, such as phishing and counterfeit websites, using survey methods. We are also evaluating reactions to possible new ways of providing protection and assistance. Results. a) Physical exercise programs are popular with the elderly and effective for increasing levels of activity, but not motivating in themselves. Some commercial games are more entertaining, but do not provide medically-recommended exercises. Our designed games with approved movements were welladopted, especially with an added problem-solving dimension. b) Social networking is popular with the elderly and can help reduce feelings of isolation. Ambient interaction devices can potentially be successful to facilitate background displays of a variety of information, such as arriving messages. Tangible objects provide a simple way of signalling a desire for contact from others, without the need to use a more complicated device. Camera-based facial expression recogni- tion is effective for tracking mood change in the elderly, and can be used as a basis for automatic requests for assistance. c) Internet insecurity is a serious issue for the elderly, but can in principle be addressed with carefully designed intervening technologies. Conclusions. Technologically-enhanced ambient environments in the homes and meeting places of elderly users can facilitate and motivate healthy exercise, social interaction and to some extent allay concerns about safety and privacy while using internet-based services. We must provide the elderly with responsive spaces, not complex devices.

\section{B1-02}

\section{Potential of Accessible Website for Elderly - Case Study Research}

\section{Željka Car, Matea Žilak, Ivana Rašan}

Faculty of Electrical Engineering and Computing, University of Zagreb, Croatia

Digital accessibility refers to a website, mobile application or electronic document that can be easily navigated and understood by a wide range of users, including those who have visual, auditory, motor or cognitive disabilities. Properly designed web and mobile applications possess great potential for removing barriers to communication and interaction that many people face in the digital world and may significantly contribute to the well-being of particularly elderly by offering new possibilities and services not only for active participation in the society, but also for supporting living independently as long as possible. The "Accessible Website for Persons with Disabilities" project is a product of long multidisciplinary cooperation between the University of Zagreb FER and Croatian Regulatory Authority for Network Industries (HAKOM). The project includes several NGOs of persons with disabilities (PWD), students from technical field from Zagreb and Dubrovnik, middle-school students and elderly from retirement homes. The overall research goal is to analyze specific needs of PWD regarding web accessibility in the country and to implement open and innovative solutions for their digital inclusion. One of the results is the accessible web prototype (usluge.ict-aac.hr/pristupacni-web-2) with the purpose of providing information on telecom operators' offers and benefits to young people, PWD and elderly. The prototype implements different accessibility features and presents the accompanied by corresponding method for developing accessible website that is distributed publicly to help developers and designers in their effort of accessible website development. Prototype evaluation was performed in several iterations 
and representatives of elderly from retirement homes also took part in this process, the oldest participant was 89 . Finally, total of $61.3 \%$ of respondents state that all accessibility options are fully implemented while $34.2 \%$ state that accessibility options are partly implemented. Part of the research performed in collaboration with the representatives of elderly focusing on those living in a nursing home shows that most of the elderly who use ICT need additional support mostly to communicate with the family and friends. This collaboration discovered new insights on what the elderly really expects the most from the digital world, as well as new ways of engaging students of technical studies in improving digital inclusion of elderly. So new course named "Application of technical knowledge for socially useful purposes" is starting at FER where students will apply their knowledge to provide support also for elderly.

\section{B1-03}

\section{Determining the Parameters of Healthy Ageing Using Automated Neuron-Level Analysis of the Laminar Structure of the Human Brain}

\author{
Andrija Štajduhar', Goran Sedmak², Miloš Judaš \\ 'Andrija Štampar School of Public Health, School of Medicine, University of Zagreb, \\ Croatia \\ ${ }^{2}$ Croatian Institute for Brain Research, School of Medicine, University of Zagreb, Croatia
}

Introduction and objectives. The ageing of the human brain is protracted process characterized by the loss of neuronal elements such as synapses and neurons. One of the most common disorders of the brain in older age are various types of dementia. The hallmark of dementia is accelerated loss of neurons which leads to the loss of many cognitive functions. In order to determine the severity of neuronal loss in different types of dementia it is necessary to precisely quantify the number of neurons in different life periods and disorders. The goal of this research is the development of novel computational methods for automatic and objective analysis of human brain at neuron level in order to achieve objective estimation of parameters of healthy and pathological brain changes as result of ageing. Methods. Current golden standard in quantification is stereology for which, in order to produce reliable number, many criteria need to be met (e.g. section randomization, tissue sectioning, etc.). Even when all prerequisites of stereology have been met, the estimate produce by it can vary $\pm 20 \%$ from real value. As many differences between "normal" and "pathological" brain fall within the $20 \%$ it is difficult to asses the real influence of neuronal loss in dementia. Machine learning methods based on tree ensembles and deep neural networks were used to estimate density of neuronal populations and predict neurons' layer within the cortex. Results. In order to obtain more precise results about neuronal number in human brain we developed novel program for automatic cell counting, phenotyping and cortical layer segmentation. The proposed solution uses deep neural networks to reliably recognize all neurons on the histological slides. The advantage of such approach is the objectivity of counting (i.e. computer always recognize the same set of neurons), reduced estimate error (all neurons on the slide are counted instead of small proportion), and reduced influence of brain anisotropy (i.e. over or under representation due to the spatial tissue properties). Through performance measurements it was shown that the machine learning model's output agrees with manual labels in the same amount as they agree with each other, thus creating results indistinguishable from that of human experts. Conclusions. Quantitative analysis of neuronal distribution and exploration of its underlying principles resulted in novel framework and methodology for cellular-level investigations of brain tissue. The use of our approach provides more reliable data about neuronal population during healthy ageing and thus providing better baseline for analysis of neuronal loss in many forms of dementia. 


\section{Poster presentations}

\section{B1-P1}

\section{Smart Home - a Solution Compatible with the EU Home-Based Care Priority?}

\author{
Mateja Banović', Maja Banadinović2, \\ Dorja Vočanec ${ }^{2}$, Aleksandar Džakula² \\ ${ }^{1}$ Health Centre Zagreb West, Zagreb, Croatia \\ ${ }^{2}$ Andrija Štampar School of Public Health, School of Medicine, University of Zagreb, Croatia
}

Introduction and objectives. Population ageing has become one of the leading challenges of modern health care systems. Chronic patient care, which greatly refers to the elderly, represents an increasing burden for health care. Combining that approach with modern technological advances, smart home care solutions emerge. Solutions like these are based on IoT networking technologies, wireless integration of parameters received from sensors and automated data transfer. The aim was to analyse compatibility of the smart home care solutions' concept with EU home-based care priority, on the example of Croatia. Methods. SWOT analysis of the elderly smart home care model was conducted. It determines strengths, weaknesses, opportunities and threats regarding possible implementation of this model in Croatia. Results. Smart home care may support independent living and reduce burden on the health care system. It enables both formal and informal carers to provide more specific and more effective care. 24/7 monitoring improves care making home a setting for the reassurance of continuity and comprehensiveness of care. It respects patientcentred and patients as a partner approaches. The level of population and technology readiness for smart home care solutions is still relatively low and limited. At the moment, the most developed seem to be sensors for monitoring daily living and chronic health conditions. Integration into existing healthcare system is also a complex challenge. Conclusion. As the growing elderly population seeks to age at familiar settings, smart home technologies can be a potential solution to support home-based care while reducing chronic care burden on the health care systems. Remote monitoring and communication with carers via existing infrastructure could allow elderly patients to remain in their homes whilst still being cared for. Digital literacy, financial accessibility, immature technology, privacy loss and internet availability in remote/rural areas as the biggest downsides will need to be considered carefully. There is a clear need for a proof of evidence study to demonstrate the software integration possibilities.
B1-P2

\section{Modern Technologies and Devices for Remote Health Monitoring in Elderly Healthcare}

\author{
Jelena Jakab, Nikola Volarić, Luka Malenica, \\ Ljiljana Trtica Majnarić, Aleksandar Včev \\ Faculty of Dental Medicine and Health, Josip Juraj Strossmayer University of Osijek, \\ Croatia
}

Introduction. Increased life expectancy along with the decreasing birthrate globally will result in a large aging population in the near future. Older age is often accompanied by numerous chronic diseases such as cardiovascular and lung diseases, stroke, diabetes and cancer, causing high mortality and strong impact on quality of life such as long-term diabetes-related complications. This unfavorable trend creates a significant socio-economic burden and increases costs associated with health care services. Therefore, enabling affordable new strategies and technologies for providing better health care is urgently needed. Emergency situations in elderly require immediate medical interventions and can result in prolonged hospital stay, all of which could be avoided or prevented by the continuous monitoring of the physiological parameters and activities. Remote health monitoring options: Remote health monitoring offers real-time monitoring, collecting and analyzing of physiological data collected by un-obtrusive sensors in a way that doesn't interrupt daily activities of normal life. Smart home platforms allow people to remain in their home environment where physiological condition and motions are monitored by non-invasive low-cost sensors which are in communication with remote healthcare facilities. Additionally, modern smartphones are equipped with a number of sensors that can be used to measure several health parameters, analyze them, display on the phone and transmit to a distant healthcare facility. Quantitative assessment of cognitive and physical health, daily activities, gait patterns, and vital signs can be useful for early detection of a potential health problem. Diabetes monitoring: Diabetes-related complications cause kidney failure, lower limb amputations, and blindness. Optimal care for patients with diabetes often lack key health information necessary for tighter diabetes control which would result in more effective therapeutic strategy. That is the reason for a recent boost in the development of glucose sensors with effective continuous sensing technology. Additionally, the broad spectrum of artificial intelligence approaches for prevention, detection and treatment of diabetes are currently being developed in order to improve glucose control and reduce hypoglycemic episodes. Conclusion. The 
primary purpose of remote health monitoring is to allow people to live uninterrupted and active lives with continuous surveillance of their physical well-being. The improvements in data storage and processing allow a low-cost solution for screening, early diagnosis and predictive algorithms development.

\section{B1-P3}

\section{SEFAC App - Tool to Support a Better Health}

\author{
Mihaela Marinović Glavić', Vanja Vasiljev', Lovorka Bilajac', \\ Tomislav Rukavina', Oscar Zanutto ${ }^{2}$, Stefania Macchione ${ }^{3}$, \\ Anastasios Rentoumis ${ }^{4}$, Athanasia Karanasiou ${ }^{5}$, Tamara Alhambra ${ }^{6}$, \\ Jorge Garcés-Ferrer ${ }^{6}$, Elena Curtopassi' ${ }^{7}$, Nigel Clark ${ }^{8}$, \\ Anthony Polychronakis ${ }^{9}$, Siok Swan Tan ${ }^{10}$, Ellen Rouwet ${ }^{10}$, \\ Sophie Korenhof ${ }^{10}$, Hein Raat ${ }^{10}$

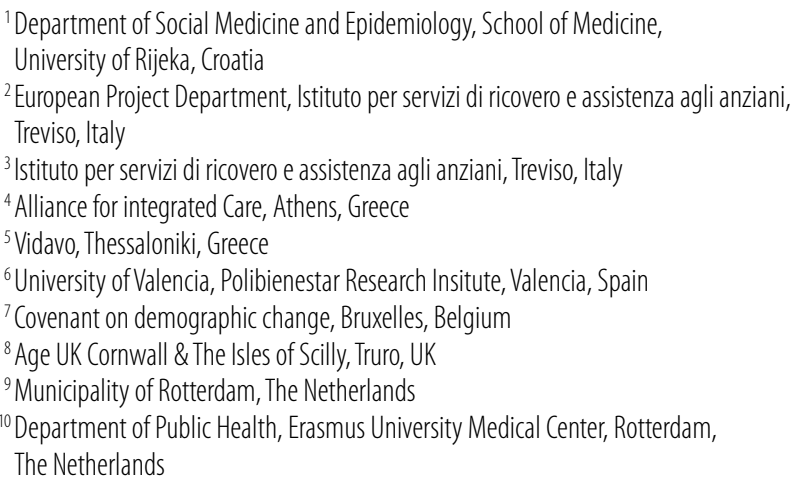

Introduction and Objectives. The expansion of novel technologies and ICT solutions can serve for the improvement of population health when used appropriately. The main challenge is digital literacy among population, especially older persons. Older persons can benefit by using new technologies and ICT solutions in many aspects (monitor of heart rate, activity time, calories, create and maintain social networks). The SEFAC project supports the population health with specifically developed ICT solution for the improvement of lifestyles in order to reduce the burden of major chronic diseases and to increase the sustainability of health systems. Participants and Methods. The SEFAC project is developed for the improvement of lifestyles of citizens $(+50)$ that are involved in 7 -week program based on the mindfulness approach. The participants were given the opportunity to use SEFAC App on their smartphones or tablets. The main goal of the SEFAC App was to enable participants to use SEFAC knowledge even after the face to face workshops ended. Results. The SEFAC app is available in English, Dutch, Italian and Croatian and it is a part of the implementation phase in four pilot sites of the
SEFAC project (Cornwall, Rotterdam, Treviso and Rijeka). The results of Rijeka pilot site implementation will be shown - creation of an user account, installing the App on the devices and showing features that SEFAC App offers (coaching and motivation, monitor nutrition and physical exercise, manage dietary/ physical activity goals, mindful bell, mindfulness practices) in order to prevent, delay, slow down or even reverse the progress of a chronic disease(s), in line with their personalized dietary and activity goals. Conclusion. The benefit that the SEFAC App offers is the possibility to empower users for pro-active self-health management making it an excellent tool for supporting and encouraging participants in a service of a healthy aging with novel technologies.

Acknowledgement: This research is part of the project '738202 / SEFAC' which has received funding from the European Union's Health Programme (2014-2020).

\section{B1-P4}

\section{Information and Communication Technologies in Service of Improving and Maintaining Mental Health in Ageing Society}

Hrvoje Mlinarić

Zagreb, Croatia

Introduction and Objectives. Burdens of ageing like chronic diseases, cognitive and social problems may result in mental health issues. Information and Communication Technologies (ICT) might offer solution for this challenge. This review gives information regarding utilization of various ICT concepts in health care and everyday life of the elderly and how it may influence their mental health. Methods. PubMed database was used for literature assessment using following keywords: Information and Communication Technologies, telemedicine, Artificial Intelligence (AI), elderly, depression and mental health. Official websites of World Health Organization (WHO) and European Union (EU) were consulted as well. Results. ICT modalities like Ambient Assistive Living Technology (AALT) based on AI help elderly to be autonomous and live a physically and socially active life. Social isolation prevention may reduce the risk of depression in elderly. Several of the top 25 most influential EUFunded ICT projects for Active and Healthy Ageing are developing cloud based cognitive training tools, fall prevention solutions, continuous care platforms and robotic systems with great potential as smart home assistance and as a part of social care for people 
with dementia through loneliness and isolation prevention. Insomnia is a significant factor of mental health issues of the elderly. Internet-based cognitive behavioral therapy programs and sleep technologies for remote monitoring of CPAP machines rehabilitate elderly with insomnia and improve their mental health outcomes. Initial phases of Agile Co Creation for Robots and Ageing (ACCRA) project designed to be tested in mobility, daily life and socialization support recorder a good overall attitude towards robotic technology and a great interest by the elderly participant to be actively involved in the development of tools based on their needs. Furthermore, different modalities of assistive technology, telecare and telemedicine have shown to be beneficial for Alzheimer disease patients and their caregivers through avoiding stress and depression. However, there are articles that emphasize a concern about consumer health information technologies regarding patients with multiple chronic illnesses. Data they track (i.e. diet or glucose levels) could be emotionally charged and may lead to low adoption of such tools. Conclusion. Enthusiasm for ICT innovation adoption is increasing and there are different technologies that have shown to be beneficial for mental health outcomes of the elderly. Although there are concerns about adoption of some types of ICTs, future improvements might help ICT utilization in service of healthy ageing.

\section{B1-P5}

\section{The Role of Standardized Diabetes Data Set in Improving the Quality of Care at Primary Care Level}

\author{
Marija Švajda' , Valerija Bralić-Lang ${ }^{2,3}$, Zrinka Mach ${ }^{4}$ Tamara Poljičanin ${ }^{1}$ \\ ${ }^{1}$ Croatian Institute of Public Health, Zagreb, Croatia \\ ${ }^{2}$ Private Family Physician Office Affiliated to the Department of Family Medicine, \\ School of Medicine, University of Zagreb, Croatia \\ ${ }^{3}$ Association of Teachers in General Practice/ Family Medicine, Zagreb, Croatia \\ ${ }^{4}$ Diabetes Patients Associations, Zagreb, Croatia
}

Diabetes has become a major public health problem last decades because of the high prevalence and consequences it has on individuals and society (1). The prevalence of diabetes is highest among adults over the age of 65 and a global increase in diabetes prevalence can partially be explained by the aging of the world's population $(1,2)$. Diabetes in older people also has numerous specificities in care and potential threats associated with treatment. To enable patients the highest possible quality of life in their third age, it is important to postpone the development of chronic complications of diabetes. Along with the chronic care model, that could be achieved by implementing a standardized data set for diabetes monitoring, which has proven to be efficient in improving quality indicators (2-6). As a part of Chrodis + Joint Action, co-financed by the EU, the research on improving quality of care by using standardized diabetes data set (SDDS) was conducted. The intervention consisted of educating family physicians (FPs) about the diabetes registry and giving them feedback about their SDDS. Mixing methods approach (quantitative and qualitative) for acquiring data was used. Quantitative analyses enabled analyzing SDDS and availability of key quality indicators before and after the intervention. Semi-structured interviews have been used to get an in-depth insight into FPs' usage and attitudes towards SDDS.An average number of patients with fulfilled SDDS per FP increased by $52.30 \%$ from 20.2 before to 30.8 after the intervention. Significant changes in values of chosen diabetes quality indicators were not observed, because of a short period of post-education monitoring within the project. The output of qualitative part enabled better insight in data set usages and revealed the recommendations for improving SDDS and quality of care as follows: implementing albumin/creatinine index in laboratories on primary health care level, SDDS feedback to FPs, involving nurses in SDDS, reducing number of standardized data sets and number of indicators per data set, making their patient management software more user-friendly, and additional funding.FPs mostly have positive attitudes towards SDDS and they use it as reminders in regular monitoring of patients with diabetes. However, educating FPs about SDDS, and giving them feedback on their work, might further contribute to better monitoring of patients with diabetes. Other possible improvement areas which might additionally improve SDDS and quality of care are ensuring monitoring of major quality indicators, higher involvement of nurses, optimization of patient management software, and additional funding. 


\section{Invited lecture}

\section{B2-I}

\section{Modern Urban Planning in the Service of Healthy Ageing}

\author{
Julia Wadoux \\ AGE Platform Europe, Belgium
}

"... healthy ageing is more than just the absence of disease. For most older people, the maintenance of functional ability has the highest importance. The greatest costs to society are not the expenditures made to foster this functional ability, but the benefits that might be missed if we fail to make the appropriate adaptations and investments. The recommended societal approach to population ageing, which includes the goal of building an age-friendly world, requires the transformation of health systems away from disease-based curative models and towards the provision of integrated care that is centred on the needs of older people."

Dr. Margaret Chan, in WHO, World Report on Ageing and Health, 2015

\section{Setting the scene: ageing and urbanisation}

In its 2018 Ageing Report, the European Commission said it expects that the proportion of the EU's population aged 65 and up will rise from 19\% to $29 \%$ by 2070 , while the segment aged 80 and over will increase from $5 \%$ to $13 \%$. At the same time, $80 \%$ of older people already live in urban areas.

Demographic shifts and urbanisation have created important challenges that need to be addressed to ensure a good quality of life for all generations, while reducing inequalities and combating social exclusion. As populations age, pressures and challenges on health and wellbeing both mount and shift and policy makers are increasingly concerned about the growing burden on health systems in general and long-term care in particular.

\section{Fostering healthy ageing through age-friendly environments}

According to the World Health Organization (WHO), physical and social environments are the key determinants of whether people can remain healthy, independent and autonomous long into their old age.
There are eight domains where this comes into play: transportation; outdoor spaces and built environment; housing; community support and health services; civic participation and employment; social participation; respect and social inclusion; and information and communication.

Improving these domains will help make cities more age-friendly. Such environments allow everyone to grow and get older in better health and to remain active and autonomous for longer. This contributes to lowering the gap between life expectancy and healthy life expectancy, which remains about 15 years for men and 17 years for women (EU28 - Eurostat). This means we spend on average between 15 and 17 years of our later life with a disabling health condition.

\section{Challenges faced by older persons in cities}

Moving from a patient approach towards a citizenship narrative is therefore key to have a comprehensive approach of older people's life. While it is important to consider the access and availability of care and health services, this is not enough. There are several challenges older people face and which impact their health status:

- loneliness and isolation: according to recent findings of the Generations and Gender Survey, loneliness was common among $30 \%$ to $55 \%$ of older people in Central and Eastern Europe and 10\% to 20\% in Northwestern Europe;

- living in place: most people prefer to continue living in their own home and "the majority of older people will continue to prefer to live in communities with a mix of ages" (Buffel, Handler, Phillipson, 2018);

- social exclusion: while material deprivation is decreasing, inequalities are rising. This covers many different issues including the impact of gentrification in some urban centres as well as energy poverty;

- accessibility: is key to foster autonomy and enable a proper mobility and access to goods and services for everyone;

- ageism and negative stereotypes: they have harmful effects on the health of older adults; research shows that older adults with negative attitudes about ageing may live 7.5 years less than those with positive attitudes (Levy et al).

In a nutshell, urban planning can be a strong tool to enhance healthy ageing and "enable older people to be and to do what they value most" (WHO, 2020), this requires to:

- create a positive view on ageing so we all can look forward to a positive future in later life and move 


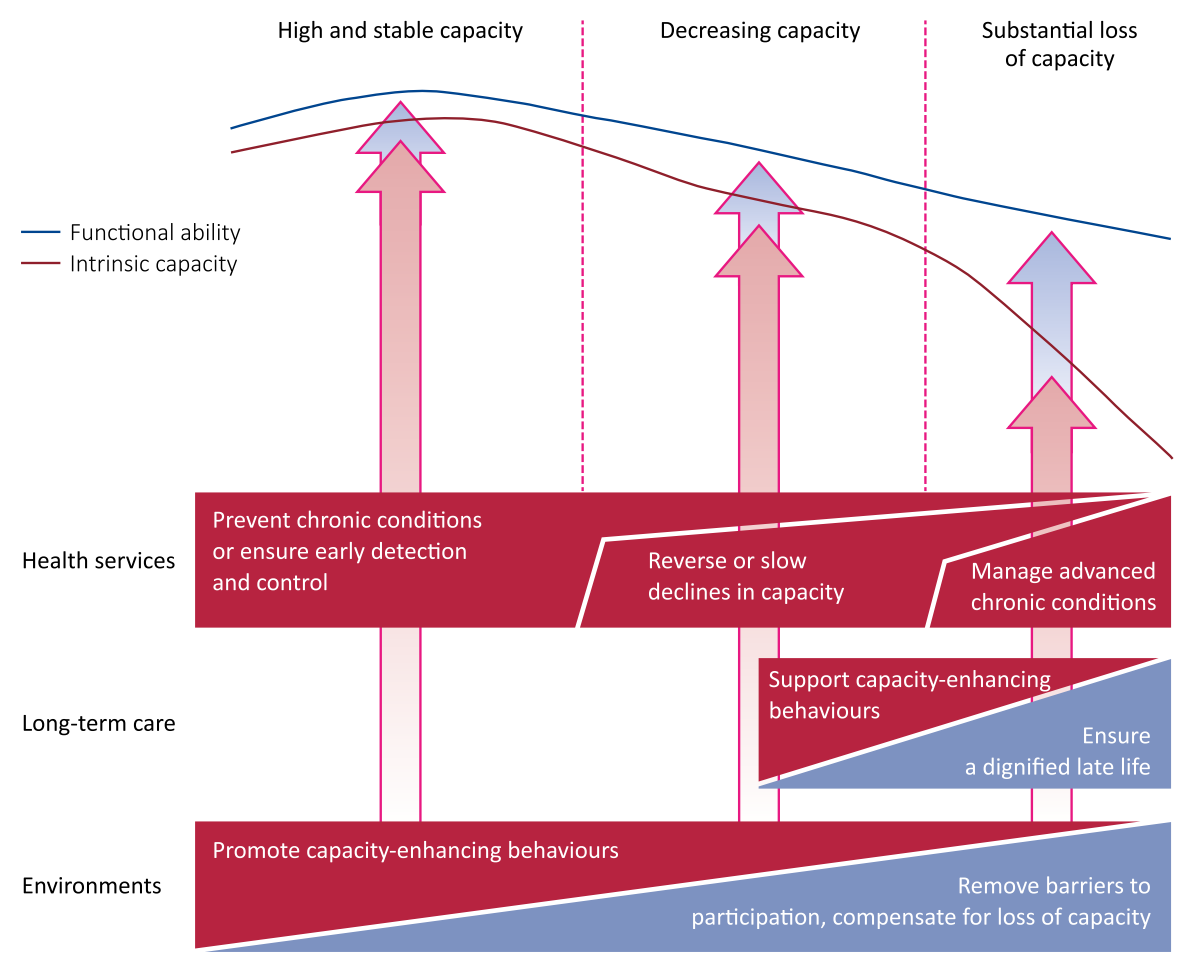

Source: WHO, World Report on Ageing and Health, 2015

A PUbliC-HEAlth FRAMEWORK FOR HeAlthy Ageing opportunities For PUbliC-HEALth ACTION ACROSS THE LIFE COURSE

from the emotional-alarming calls that a 'Silver Tsunami' is coming to an empathetic humanright based movement of society for all ages;

- build a narrative based citizenship approach: ageing is not only about a medical and care approach;

- enable active involvement citizens, including older persons, in the development and implementation of policies and activities in order to respect lifestyle choices, needs and preferences;

- organize and plan cities for persons of all ages: fostering solidarity between generations is key for a sustainable future;

- break the silos between the different municipality department and mainstream ageing issues;

- ensure a strong political support with a fair funding;

- develop an ecosystem enhancing close collaboration between various stakeholders (citizens, re- searchers, policy makers, businesses): everyone has a role to play;

- provide a proper problem analysis, with meaningful data, and evaluation of the solutions implemented: this is key to learn lessons and move forward while ensuring continuity when needed.

\section{R E F E R E N C E S}

1. WHO. World Report on Ageing and Health, 2015 - https:// www.who.int/ageing/publications/world-report-2015/en/

2. European Commission, 2018 Ageing Report - https://ec.europa. eu/info/publications/economy-finance/2018-ageing-reportunderlying-assumptions-and-projection-methodologies_en

3. Buffel T, Handler S, Phillipson C, Eds. Age-Friendly Cities and Communities - A global perspective, Bristol, UK: Policy Press; 2018. 


\section{Oral presentations}

\section{B2-01}

\section{Social Design for Healthy Ageing}

Ivana Fabrio

School of Design, Faculty of Architecture, University of Zagreb, Croatia

Social design is a fast-growing field of design in recent years, tackling many issues across all facets of human life. Designers, who have traditionally been defined as problem solvers, have broadened their scope of impact towards developing systems and strategies as well as objects, serving the needs of undeserved communities. Therefore, students, who have grown in age of economically turbulent times are focusing their ambitions towards humanitarian work that helps solve complex social issues. These involve critical issues such as fragility of health, ageing, lack of natural resources or educational services and so on. Social design uses methods of participatory practice in collaboration with social enterprises, NGOs, foundations, corporations, and governments, with a goal to generate long-lasting solutions. Participatory design practice includes design research methodologies as well as participation of all the stakeholders throughout the whole process, from problem defining to solution and implementation. The methodology suggests a pattern of steps to define a problem, generating ideas and translating ideas into value. These processes include attributes of design thinking, that consist of five crucial phases, each aiming at human centered solutions, and including practices of ethnographic research and generative processes such as divergent thinking. The ideas are then tested through rapid prototyping and validated through a process of convergency to create consensus necessary from all stakeholders in order to execute a solution that results in real progress. The presentation will show step by step appropriation of this participatory methodology in social design projects that consider community members as partners in all aspects of the design process. The focus will be on projects that aim at empowering the ageing community through various approaches and solutions, focusing on intergenerational interactions and transactions that are materialized through urban, spatial and product solutions. Two projects will be shown as an example of social design aimed towards elderly population: 1. project LAND - is based on the daycare center that connects elderly people with kindergarten kids through a system of participating activities, that include architecture and product design as tools. LAND is a collaborative project between students of Architecture and School of Design and has won innovation award at the International FINSA Award 2018. 2. project „Volunteers in the park“- is a part of „Živjeti zdravo" national program, which is a collaboration of six faculties within University of Zagreb, and was organized by The School of Public Health Andrija Štampar

\section{B2-O2}

\section{Inclusive Design (for Ageing Population)}

\author{
Sanja Bencetić
}

School of Design, Faculty of Architecture, University of Zagreb, Croatia

Global and local population ageing severely influence common image of average person, and traditional methods of addressing human needs through design. Contemporary principles and methods presented by inclusive design could answer these changes.

Basic inclusive design principles and methods are presented by review of four ground-breaking past projects. Following is the presentation of several original projects applying these principles and methods, with results, discussion and conclusion.

\section{B2-O3}

\section{Green and Healthy Cities - Environmental Exposures and Urban Design for Healthy Longevity}

\section{Agnieszka Olszewska-Guizzo}

NeuroLandscape Foundation, Singapore

In the face of ongoing urbanization and aging of the society, we can easily conclude that soon enough most older adults will live in urban environments. There is a consensus between the researchers and professionals that the urban living is stressful and can cause mental health issues, what is more, aging itself causes risk of developing mental health disorders. It is then necessary to investigate the spatial and environmental determinants of mental health and well-being in cities, targeting different age and social groups (from the antenatal to elderly phase of life). In this presentation I will discuss possible mechanisms of environmental exposures and mental health outcomes, with a focus on green spaces network within the city. I will present the current research status and gaps in knowledge to address the mental health issues, the limitations and 
opportunities of evidence-based urban design, as well as the good examples from Europe and Asia.

\section{B2-04}

\section{Healthy Urban Planning} for a Better Future for Healthy Ageing - Healthy Urban Planning, the Croatian Healthy Cities

\section{Network, and the City of Vinkovci} (Croatia)

\section{Mandica Sanković \\ City of Vinkovci, Croatia}

Healthy urban planning is the attainment, preservation and improving health of all users of space through urban planning, urban planning, organization of the entire space of towns / settlements / cities. According to WHO terminology, healthy urban planning includes: urban planning, design, construction, supervision, maintenance. Urban planning is a multidisciplinary science related to: construction of new cities and settlements and study of their development, structures and life in the past, present and future. Urban planning as a multidisciplinary science includes: geography, economics, sociology, ecology, medicine, philosophy, law, transport and many other sciences. The principles of sound urban planning are based on the principles of healthy city: equality, social cohesion, multidisciplinary cooperation and community involvement. The impact of healthy urban planning is monitored through healthy urban planning indicators, which include 37 indicators in five areas: A-Health indicators, B-health indicators, $\mathrm{C}$-environmental indicators, D-socioeconomic indicators and E-general data for the observed population. The Croatian Healthy Cities Network and the City of Vinkovci, through Vinkovci Healthy City project, implement measures aimed at improving population health by using, among other, healthy urban planning projects based on science. Improving health using healthy urban planning is very effective, but it demands holistic intersectoral work, education and political support. Healthy urban planning holistically determines activities for sustainable development, a better future, a healthy lifestyle from an early age, and creates the precondition for healthy and independent aging. The term and the activities related to healthy urban planning should be implemented into legal acts and at all phases of the whole process schooling and work. In that case, the implementation of healthy urban planning would be mandatory and a sustainable development would become more attainable and sustainable development, healthy and independent aging more achievable. The Ottawa Charter on the Promotion of Health (1986), the Health for All Strategy, emphasizes the need to promote health, noting that only the health sector is responsible for the health of society. „Healthy city is a city for all its citizens, inclusive, supportive, socially sensitive, and capable of fulfilling different needs and expectations of its citizens". 


\section{Poster presentations}

\section{B2-P1}

\section{Urban Planning for Healthy} Ageing: The Example of Park Planning in the City of Zagreb

\author{
Martina Jurjevići, Maja Lang Morović, \\ Sanja Musić Milanović2,3, Mirjana Kujundžić Tiljak ${ }^{4}$ \\ ${ }^{1}$ City Office for Urban Planning, Construction, Housing and Utilities and Transport, \\ Zagreb, Croatia \\ ${ }^{2}$ Division for Health Promotion, Croatian Institute of Public Health, Zagreb, Croatia \\ ${ }^{3}$ Division for Epidemiology, Department of Medical Statistics, \\ Epidemiology and Medical Informatics, Andrija Štampar School of Public Health, \\ School of Medicine, University of Zagreb, Croatia \\ ${ }^{4}$ Division for Medical Statistics, Department of Medical Statistics, \\ Epidemiology and Medical Informatics, Andrija Štampar School of Public Health, \\ School of Medicine, University of Zagreb, Croatia
}

Introduction and objectives. Urban planning with the aim of health preservation and promotion is a rather novel technology. The literature on urban planning for healthy ageing is scarce. Still, within various national health promotion programs and projects, the need for urban planning for health in general and specifically, healthy ageing is continuously emphasized. According to the World Health Organization, by careful urban planning it is possible to support physically active lifestyles in people in their environments, increase the share of healthy population and establish spaces that enable healthy ageing. The goal of this paper is to present urban planning of one of the public surfaces in the City of Zagreb as an example of healthy ageing promotion. Methods. Residents of the Zagreb City center are in majority elderly and lack public spaces that would motivate them to go outside in the open more often, socialize and be more physically active. Due to the meaning and proximity of leading national public health institutions, the space at the beginning of Mirogojska Street with staircase leading towards Andrija Stampar School of Public Health and Croatian Institute of Public Health was selected to become a place where elderly people will be able to achieve some of the healthy ageing goals. This space however, was not used for years and has with time become unreachable posing a certain risk to the people. Architects in charge have projected this area to be the future meeting point in nature where people can be both physically active but also find their peace from everyday life while applying minimal interventions into the current architecture. Results. The healthy green city area was designed with minimal interventions. The impressive staircase was renewed. The plant stock was kept to the fullest extent possible. The landscaping is dominated by publically lighted walking path at which end points there are places to sit, relax and socialize. The herb garden, dominated by lavender and rosemary, follows higher part of the trail. This way, the park also ensures an olfactory and visual sensory experience. An additional area of this park is a plateau designed to gather residents for events. The space next to the plateau has been left behind for the future monument of Slobodan Lang, a prominent physician and humanitarian, who spent his life working at both Andrija Stampar School of Public Health and Croatian Institute of Public Health. Conclusion. With multidisciplinary work for health and by taking people's health needs into account in urban planning, we can contribute to the rise of healthy people and promote, among other, healthy and quality ageing.

B2-P2

\section{The Need for Early Evaluation of the Implementation of the Re-orientation Programme for Home Assistance Services to} Ensure Its Sustainability

\author{
Jadranka Mustajbegović \\ WHO CC Occupational Health in Croatia, Croatia \\ Croatian Academy of Medical Sciences, Croatia
}

Introduction and Objectives. The EU project Zaželi (engl. Wish ) of the Croatian Ministry of Labour and the Pension System is re-orientation training programme for women in the Home Assistance Service, an extra-institutional form of the protection of older people. It covering unemployed women over 50 years of age, who fall into the category of hard-to-employ workers and it is being implemented polycentrically in different parts of Croatia. The program lasts for 30 months and the first six months are scheduled for the education and training of women because they need to be educated to do these jobs. EUSUZ - The Centre for promotion of European standards in health care provides education and re-orientation training programme in $\mathrm{Za}$ greb (Zaželi za Zagreb; engl. Wish for Zagreb). According to the criteria of occupational medicine, it is a sensitive group of female workers who are engaged in work with also very sensitive users of their services: the elderly who need help. The circumstances in which the home assistance services take place are very complex and demanding. Therefore, the objective was to recognize the most prominent organisational and psychosocial factors that arise from the above circumstances, 
which can significantly jeopardize or even disable the achievement of the noble goals of this project. Methods. Based on first-hand experience in working with their customers, 54 trainees were asked: 1 . List up to 3 interesting and enjoyable experiences with your customers; 2 . List up to 3 of the most challenging problems you face when working with customers; 3 . Do you think you need additional professional help to resolve any of the issues you have identified? Results. There are 3 groups of issues identified that require immediate intervention by those responsible for project implementation: hard physical effort- hard labour; users' wrong perceptions of home assistance - what services they can expect; and considering the age of women who are in training- their working or health capacity are often reduced. Conclusions. To bridge the major challenges, the solutions can be: at the beginning bring the customer's household to a "zero" condition with a cleaning service - then the home assistance service can maintain afterwards; a written "agreement" with the customer and with the women in home assistance service; further training of women in home assistance service and their familiarisation with social security and health systems.

\section{Invited lecture}

B3-I

\section{The Role of IT Companies in the Innovation Practices for Healthy Ageing}

Rain Laane

Estonian Health Insurance Fund, Estonia

Abstract not sent.

\section{Oral presentations}

B3-01

\section{Health, Social and Financial Consequences of the Application of AI, ML and Digital Health - Going Far Beyond the Traditional Healthcare System}

Stjepan Orešković ${ }^{1}$, Mario Ravić ${ }^{2}$

1 Department of Medical Sociology and Health Economics, Andrija Stampar School of Public Health, School of Medicine, University of Zagreb, Croatia ${ }^{2}$ Ericsson Nikola Tesla d. d., Zagreb, Croatia

We will employ the case of the ageing population to make an inquiry into the nature and causes of the transition of the healthcare system and health-related industries to the value-based healthcare model. The concept is based on the idea that purchasers of health care such as government, public and private employers, and individual consumers and single payers, multiple payers, individuals hold the health care delivery system accountable for the best possible quality of care. Increasing cost burden from chronic health conditions and aging population is the key driver for digital health solutions such as $\mathrm{m}$-Health applications, RPM devices, telehealth platforms, and PERS. Furthermore, favorable reimbursement policies towards clinically relevant digital health applications. There are significantly expanding care delivery models beyond physical medicine to include behavioral health, wellness therapies, dentistry, nutrition, and prescription management - critical areas of care for the 65+ population. Healthcare is at a tipping point for leading tech 
companies (Amazon, Apple, IBM, Google, and Microsoft) developing technologies suited for healthcare industry use cases. Applications for healthcare are gaining prominence as tech moguls are aggressively developing applications such as quick medical scribes and transcription speech-based guided interactions to vetted clinical use cases such as elderly care and chronic condition management. The key "tool/instrument" of transition is $\mathrm{AI} / \mathrm{ML}$ across clinical and non-clinical arena bolstering the growth of healthcare space. AI/ ML will begin to see fruition, particularly in the risk analytics applications for the aging population. The application of digital health will continue to go far beyond the traditional system and empower individuals to be able to manage their own health what is of critical importance for self-dependent aging population. It is expected that digital health tech catering to out of hospital settings, including services for the 65+ population will grow by $30 \%$ crossing $\$ 25$ billion markets globally. Application of AI/ML algorithms in healthy aging is highly dependent on data availability and integrity. Ericsson Nikola Tesla has developed solution "Smart Habits" for independent living of elderly people which by collecting information from sensors learns the patterns of daily living using ML algorithms and provides actions on behaviors different than usual. Integration of various data sources from activity to machine generated vital parameters readings in addition will serve as basis for prediction modeling in chronic disease management.

\section{B3-O2}

\section{Humanoid Robots as Assistants in Healthy Ageing}

\author{
Dora Matić, Ivan Bejić, Ivan Buterin, Zvonimir Jukić, Filip Kozlik, \\ Vladimir Slošić, Ivan Hrabar, Frano Petrić, Zdenko Kovačić
}

Faculty of Electrical Engineering and Computing, University of Zagreb, Croatia

Introduction and Objectives. Medical practice worldwide is facing a problem with too few medical staff, given the growing number of older patients and their more demanding needs. Therefore, caregivers are often inaccessible, overworked, and patients are more at risk. To alleviate this problem, care systems based on the use of humanoid robots have been developed. We focus on using the characteristics of a humanoid robot to implement two demonstration scenarios: (i) demonstrator and supervisor of rehabilitation exercises for hands and arms, and (ii) medical assistant in a caregiving facility. Methods. We use the abilities of the robot to recognize humans' faces and emotions, as well as the ability to communicate with smart devices (IoTbased) that offer better comfort and continuous moni- toring of the health status of elderly patients. Results. Robot's assistance to physiotherapists during execution of hand and arm rehabilitation exercises was implemented using NAO and Pepper humanoid robots. We have implemented a robot choreography for 18 exercises. While each exercise is in progress, the robot first explains to patients how an exercise should be performed, repeats those instructions and counts how many times an exercise has been repeated. It also talks to raise their motivation during exercising. The tests were done in the laboratory conditions and in the exercise rooms of the Clinical Hospital for Traumatology in Zagreb. The reactions of patients were very positive. During implementation of a medical assistant role, the focus was put on the interaction between the robot and the people around it, and the use of IoT devices had a goal to increase the quality of patient care. These new robot functionalities owing to the addition of IoT devices enabled the robot to perform the duties of a medical assistant more autonomously. This enabled the collection of patient status data (amount of sleep, physical activity, pulse). Conclusions. Assistive robotic technologies are recognized by the medical and care-giving community as being a strong support for healthy aging of the elderly population. They can help in slowing down the process of dementia, increasing the level of physical fitness and starting the process of becoming a social companion. One of the main benefits of using humanoid robots like Pepper is their ability to easily communicate with care-giving staff, which greatly speeds up the task. In addition to technical assistance to staff, the robot also offers social aspects of patient care. With its appearance and manner of communication, it can provide emotional support to patients and shortening their time through companion dialogue or play.

\section{B3-O3}

\section{Simulating and Comparing the Effects of Preventive Programmes: An Application}

\author{
Sabato Mellone', Pierpaolo Palumbo ${ }^{1}$, Beatrix Vereijken², \\ Jorunn L Helbostad ${ }^{2}$, Lorenzo Chiari ${ }^{1}$ \\ 'Department of Electrical, Electronic, and Information Engineering "Guglielmo Marconi". \\ University of Bologna, Bologna, Italy \\ ${ }^{2}$ Department of Neuromedicine and Movement Science, Faculty of Medicine \\ and Health Sciences, Norwegian University of Science and Technology, \\ Trondheim, Norway
}

Introduction and Objectives. Prolonged life expectancy and low birth rate is steadily increasing old age dependency ratio. In 2019, the ratio was $35.7 \%$ in Italy, 
the highest value in Europe. Healthcare systems will have to shift, by force of circumstances, from treatment towards prevention of age-related medical conditions. Since falls in the elderly are a major cause of morbidity and mortality, fall risk assessment tools and intervention programmes are major research topics. Nevertheless, current guidelines for fall risk assessment have relatively low performance [1] and there are few properly validated risk screening methods. The PreventIT project developed and tested the feasibility of an ICT-based personalised behaviour change intervention for preventing functional decline at older age, targeting amongst others common risk factors for falls. Feasibility and cost estimates were assessed in a multicentre, three-armed randomised controlled trial [2], including a control group, a manual- plus instructor-based intervention (aLiFE), and a mobile healthbased intervention (eLiFE). The aim of the current study is to simulate and compare the effects of the PreventIT interventions using the Italian situation. Methods. Starting from the simulation analysis reported in [1], we introduced: i) the statistics reported by the Italian National Institute of Health about prevalence, consequences, and related direct costs of falls; ii) the cost estimates for the PreventIT interventions. Results. Assuming the screening and intervention cost as direct cost of the healthcare service, the approach based on the simulation analysis in [1] would not be sustainable because of the poor performance of the risk screening tool and of costs associated with a traditional multifactorial intervention. Assuming the same efficacy for the intervention and state of the art predictivity for the risk screening tool, aLiFE would not be sustainable because of the costs associated with healthcare professionals and trainers involved in the intervention. In contrast, eLiFE, based on self-assessment and smartphone-based intervention, would be sustainable. Conclusions. Despite the limitations of this study, results emphasise the need to develop innovative solutions for empowering users to take care of their own health and function. The availability of effective digital tools, the integration with electronic healthcare records, and the integration with patient generated data will become crucial for the sustainability of healthcare services in the near future.

\section{Poster presentations}

\section{B3-P1 \\ ICT Technologies \\ as New Promising Tools for the Managing of Frailty: A Systematic Review}

Alessia Gallucci ${ }^{1,2}$, Pietro Davide Trimarchi', Carlo Abbate ${ }^{1}$, Cosimo Tuena ${ }^{3,4}$, Elisa Pedroli ${ }^{3,5}$, Fabrizia Lattanzio ${ }^{6}$, Marco Stramba-Badiale ${ }^{7}$, Matteo Cesari ${ }^{8,9}$, Fabrizio Giunco ${ }^{1}$

${ }^{1}$ IRCCS Fondazione Don Carlo Gnocchi, Milan, Italy

${ }^{2}$ Ph.D. program in Neuroscience, School of Medicine and Surgery,

University of Milano-Bicocca, Monza, Italy

${ }^{3}$ Applied Technology for Neuro-Psychology, IRCCS Istituto Auxologico Italiano, Milan, Italy

${ }^{4}$ Department of Psychology, Catholic University of the Sacred Hearth, Milan, Italy

${ }^{5}$ Faculty of Psychology, University of eCampus, Novedrate, Italy

${ }^{6}$ Scientific Direction, IRCCS INRCA, Ancona, Italy

${ }^{7}$ Department of Geriatrics and Cardiovascular Medicine,

IRCCS Istituto Auxologico Italiano, Milan, Italy

${ }^{8}$ Geriatric Unit, Fondazione IRCCS Ca'Granda Ospedale Maggiore Policlinico, Milan, Italy

${ }^{9}$ Department of Clinical Sciences and Community Health, University of Milan,

Milan, Italy

Objectives. Frailty associated with ageing is a major health issue as it encompasses functional decline, physical dependence and increased mortality risk. Recent studies explored Information and Communication Technology (ICT) as valid alternatives to manage frailty in elderly. The aim of the present review was to synthesize current evidence on ICT technologies application within the more complex assisting models of frailty in older people. Design. A systematic review of systematic reviews was conducted. Data sources included PubMed, Psychinfo, EMBASE and Web of science, considering eligible those reviews on ICT application in samples of elderly formally assessed as frail. Two independent researchers screened the records, extracted data and appraised methodological quality of reviews and studies. Setting and Participants. Frail elderly with a mean age of $\geq 65$ years in any health care or residential setting, or community dwelling elderly people. Results. Among the 764 papers retrieved, we included 2 systematic reviews. Sixteen observational cross-sectional studies and one randomized control trial were analyzed across the systematic reviews. Most of the studies defined frailty considering only few components of the phenotype, and used ICT to stratify different levels of frailty or to support traditional screening strategies. Indeed, assessment of frailty is the setting in which ICT has been mostly tested as compared to intervention. Studies did not report cost 
effectiveness evaluations of the ICT technologies employed. Conclusions and Implications. The research investigating the use of ICT in the context of frailty is still in its initial lines. Based on our results, there is no evidence supporting the ICT efficacy for the care of frailty, indeed ICT treatment strategies of frailty were slightly reported. The lack of a proper characterization of the frail condition along with the methodological limitations prevented the investigation of ICT within complex assisting models of frail older people. Future studies are needed to effectively integrate ICT in the care of frailty.

\section{Session B4: Mobile Techonoglies and Primary Healthcare}

\section{Invited lecture}

\section{B4-I}

\section{Mobile Technologies and Primary Healthcare}

Venet Osmani

Fondazione Bruno Kessler Research Institute, University of Trento, Italy

Life expectancy continues to increase owing to better quality of life and improved medical care. While the increase in life expectancy is an important achievement it tells only part of the story of the overall health of the population. Together with the life expectancy, the disease-free lifespan is also an important factor to be considered. Disease-free lifespan (known as healthspan) refers to the years of person's life spent in good health, free from the chronic diseases and disabilities of aging. However, there is some evidence to suggest that healthspan has not kept up with the increase in lifespan (1). Data from World Health Organisation shows that a global increase of 5 years in total life expectancy between 2000 and 2015 has been accompanied by only 4.6 years of healthy life expectancy (2). In fact, increasing life expectancy has increased the risk of chronic diseases including cardio-vascular, cancer and neurodegenerative diseases, where majority of these diseases are primarily manifested in older people. Considering socio-economic implications of this trend, there should be a focus shift from lifespan towards healthspan as the principal outcome and most important goal of medicine (3).

Research into extending healthspan has been tackled across diverse disciplines, from basic molecular and cellular mechanisms that drive ageing through to novel treatments and lifestyle interventions. While undoubtedly genetics play a major role there is also a vast potential for lifestyle interventions that could delay manifestation of chronic diseases and ageing-related disabilities. The focus of this work will be on the latter, describing the potential of technological interventions in increasing healthspan. A particular focus will be put on the potential of mobile technologies to administer large-scale, personalised lifestyle interventions. This work will also provide an overview of the research in this area including a review of the $\mathrm{EU}$ initiatives in healthy ageing as well as research from individual research groups. The work will conclude with a presentation of the results from the projects and initiatives in healthy ageing carried out in the Trentino region in Italy.

\section{R E F E R E N C E S}

1. Crimmins E M. (2015). Lifespan and healthspan: Past, present, and promise. Gerontologist. https://doi.org/10.1093/geront/ gnv130

2. Melzer D, Pilling L C, Ferrucci, L. (2020). The genetics of human ageing. Nature Reviews Genetics. https://doi.org/10.1038 /s41576-019-0183-6

3. Olshansky S J. (2018). From Lifespan to Healthspan. JAMA Journal of the American Medical Association. https://doi. org/10.1001/jama.2018.12621 


\section{Oral presentations}

\section{B4-01}

\section{Set Up of an Ecosystem for the Digital Transformation of Health and Care: Campania Experience}

\author{
Maddalena Illario \\ Health's Innovation Unit, Campania Region, Naples, Italy
}

Campania is a region of southern Italy, with 5.801.692 residents (ISTAT, 2019) and a growing percentage of older adults from 14.3 (2002) to 18.8 (2019), with an ageing index of 129.8 and a dependency index of 49,9 . Campania ageing population poses new challenges such as the need to switch from acute and reactive care to a proactive approach to health and to the integrated management of multiple chronic diseases, with the aim of reducing dependency and disability. Innovative, ICT-supported solutions conjugated with change management can speed up the process of change and improve the sustainability of Campania social and health system. The involvement of Campania in the European Innovation Partnership on Active and Healthy Ageing since 2013 has been stimulating and supporting the set-up of a local ecosystem to face the challenge of an ageing population with a lifecourse approach, where innovations are exploited to improve health outcomes, quality of life and sustainability of social and health services. The backbone of Campania RS has been developed according to the quadruple helix of the RS, and is rooted in the ProMIS@Campania network. It is connected to the national ProMIS network for the internationalization regional health services, and includes a referent for each one of the stakeholders of the regional health system. This core network has then been building structured connections along the 4 helix with: Universities and Research (Bioteknet District); No profit Organizations; Industry (Campania Digital Innovation Hub @Confindustria). Our ambition is connecting innovations with endusers by: $\bullet$ Increasing health equity for Campania citizens by improving sustainability through change management strategies and large-scale adoption of ICT supported services, strengthening integrated care; $\bullet$ Overcoming the boundaries of a system oriented to manage diseases, improving health promotion and disease prevention; -Strengthening the transfer of biomedical and technological innovations to the market, exploiting the integration between research, training and service provision through international collaborations, for example through the EIP on AHA, the PPI and innovative procurement; $\bullet$ Stimulating multidisciplinary and multi-actor collaborations, through living labs that are designed to fit local socio-cultural contexts. To contribute in the achievement of these objectives, Campania RS has been supporting its stakeholders to join collaborative activities at locoregional, national and international levels along 4 main directories: Disease prevention \& Health promotion; Integrated Care; Strategy and change management; New market opportunities.

\section{B4-02}

\section{EU Project for Innovative Active and Healthy Ageing - City of Zagreb}

Antonija Balenović

Health Center Zagreb - Center, Croatia

Active and healthy aging and holistic care for the elderly has been one of the most important public health priorities of the City of Zagreb since 1987, when the City of Zagreb became part of the large European network of the World Health Organization "Healthy Cities", which reflects the City's commitment to improving all policies that contribute improving health and social well-being, especially for vulnerable groups of citizens. Based on the results of a long-standing policy and overall care for the elderly, the City of Zagreb during 2016 joined European Innovation Partnership for Active and Healthy Aging (EIP on AHA), which represents a network of EU Regions promoting active and healthy aging. During 2019 the accreditation process was reiterated and the Health Center Zagreb-Center as holder and the City of Zagreb as a region confirmed the status of the Reference Site and upgraded it from one to three stars, indicating the status given to those organizations and regions that have shown excellence in development in the previous period and adopting innovative practices for active and healthy aging. This platform is a communication and information hub for all actors involved in Active and Healthy Ageing through Europe. It is the place to encourage partner engagement, promote news and events, meet and exchange ideas with peers, and look for potential partners on innovative projects. Only cooperation can lead to structural changes that go beyond the scope that an individual organization could achieve on its own, thus helping to create an environment for learning, sharing knowledge and adapting to the capabilities and realities of different regions. The improvement of the digital innovation system and the use of digital tools is an area of development that needs to be invested since there is a significant increase in the proportion of older people with multiple chronic conditions in the City of Zagreb. Digital tools and ICT also can be helpful in 
adherence to medical plans of older people living with chronic conditions and improve the quality of life and health outcomes by delivering tangible adherence approaches for patients in various disease areas. Our actions are based on a holistic approach, including enhanced self-care, personalized care, better adequacy of treatment and increased adherence to safe and effective care plans. Innovative organizational, technical and medical practices for better adherence can lead to more efficient use of resources and the efficiency of health interventions. Ultimately, it can improve the health of patients, their quality of life, reduce the worsening of the disease and avoid unnecessary hospitalizations.

\section{B4-03}

\section{Bridging the Digital Divide - ICT for Older People in Rijeka}

Jadran Mandekić

Department of Health and Social Welfare, Rijeka, Croatia

The population of Rijeka is demographically quite old. A negative population growth has been recorded over the past 20 years, while the effects of the economic crisis drove a lot of young people to leave the country. This is one of the reasons why fostering a healthy ageing environment and keeping people healthy and active as they age is at the top of Rijeka's priorities. The importance of ICT technology as an effective social engagement tool cannot be understated in today's world. This fact was clear to the City of Rijeka and so the City's Department of Health and Social Welfare and the Information Technology Department started a series of activities that jointly became known as "A society in which I am learning and feeling good". To help bridge the digital divide, in 2005 Rijeka started providing free yearly ICT courses for older people. The courses are aimed at teaching basic and advanced IT skills. Rijeka is continuously financially and logistically supporting the operation and activities of 16 Clubs for Older People. The Clubs provide an opportunity for socialization and participation in many organized and free activities. As the level of digital literacy of older people of Rijeka grew, the City started creating small Digital Centers in the Clubs and equipping them with PCs, free Internet access and other ICT equipment. In order to motivate older people to use their newfound ICT knowledge and to encourage greater social participation, the City of Rijeka created a website aimed at older people (http://penzici.rijeka.hr), where the majority of content is created by the users themselves. To encourage greater activity on the por- tal, a yearly "Top Online Pensioners" competition was organized. While it is challenging to properly motivate older people to engage in new activities and expand their comfort zone, Rijeka has learned that through education, proper means of motivation and citizen participation, older people are not only willing to participate, but are also very involved in creating new opportunities for themselves and their peers. This is noticeable through increased traffic on the website, more visits to the Clubs for older people, as well as greater overall participation in offered activities. Healthy ageing and the creation of a healthy ageing environment plays a large role in reaching many Sustainable Development Goals and therefore Rijeka is going to continue to focus its efforts in this direction.

\section{Poster presentations}




\section{Invited lecture}

\section{B5-I}

\section{Intelligent and Explainable Shareable Clinical Pathways}

\author{
David Mendes \\ Universidade de Évora, Portugal
}

Population ageing poses challenges to societies never equated. In fact, as a result of the lifepath of the current elderly, they are aging with high loads of disease in the form of multimorbidity and dependence. Such circumstances induce a high consumption of care, a huge pressure on the health and social safety system and, at the same time, an urgent need to change the organizational models of provision and care due to the adjustment to the previous circumstances. We propose that AI (Artificial Intelligence) comes to the rescue. In order to enable automated clinical reasoning (aCR) both an innovative care model and a supporting infrastructure must be developed and put in place.

The proposed care contract entails all the levels of care from self to highly specialized care and can be pictured like this:
We are presenting a knowledge infrastructure to enable the aCR in a timely and responsive manner. The knowledge base is a "Digital twin" of the health and care conditions throughout the lifespan that has some aspects specific for our model representation. ${ }^{2}$ Namely, two very important and distinctive characteristics that look obvious to any clinician but are not evident for any IT implementer:

1. It maintains a two-fold time series for representing the health and care history and events: It has both a historical and a "current events" time series and both are considered for the aCR to be performed accurately. In the first all the health condition antecedents are collected by a forefront running agent when the personal process is created and several and distinctive knowledge sources are used from the different institutions involved in our blockchain network. In the second a continuous stream of events are monitored for the adequate response generation and knowledge structure enrichment coming from the sensors available in the ALIE (Assisted Living Intelligent Environment).

2. A carefully crafted information fusion layer:

The sensors that continuously gather vital signs information are low powered artifacts that function in the edge computing layer, very close to the person, but they must have some reasoning abilities. They provide features to the (automat-

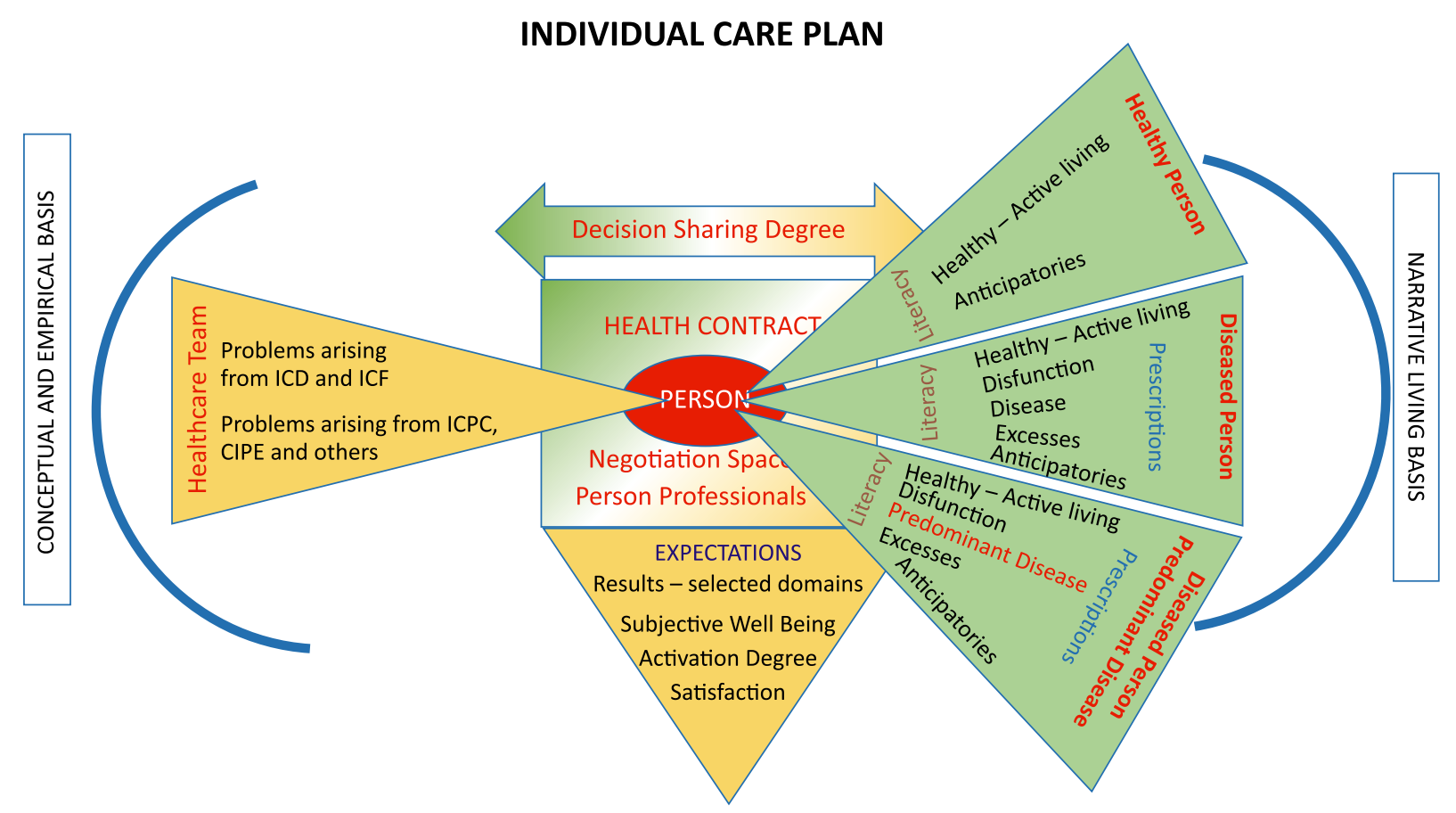

Figure 1. Individual Care Plan 


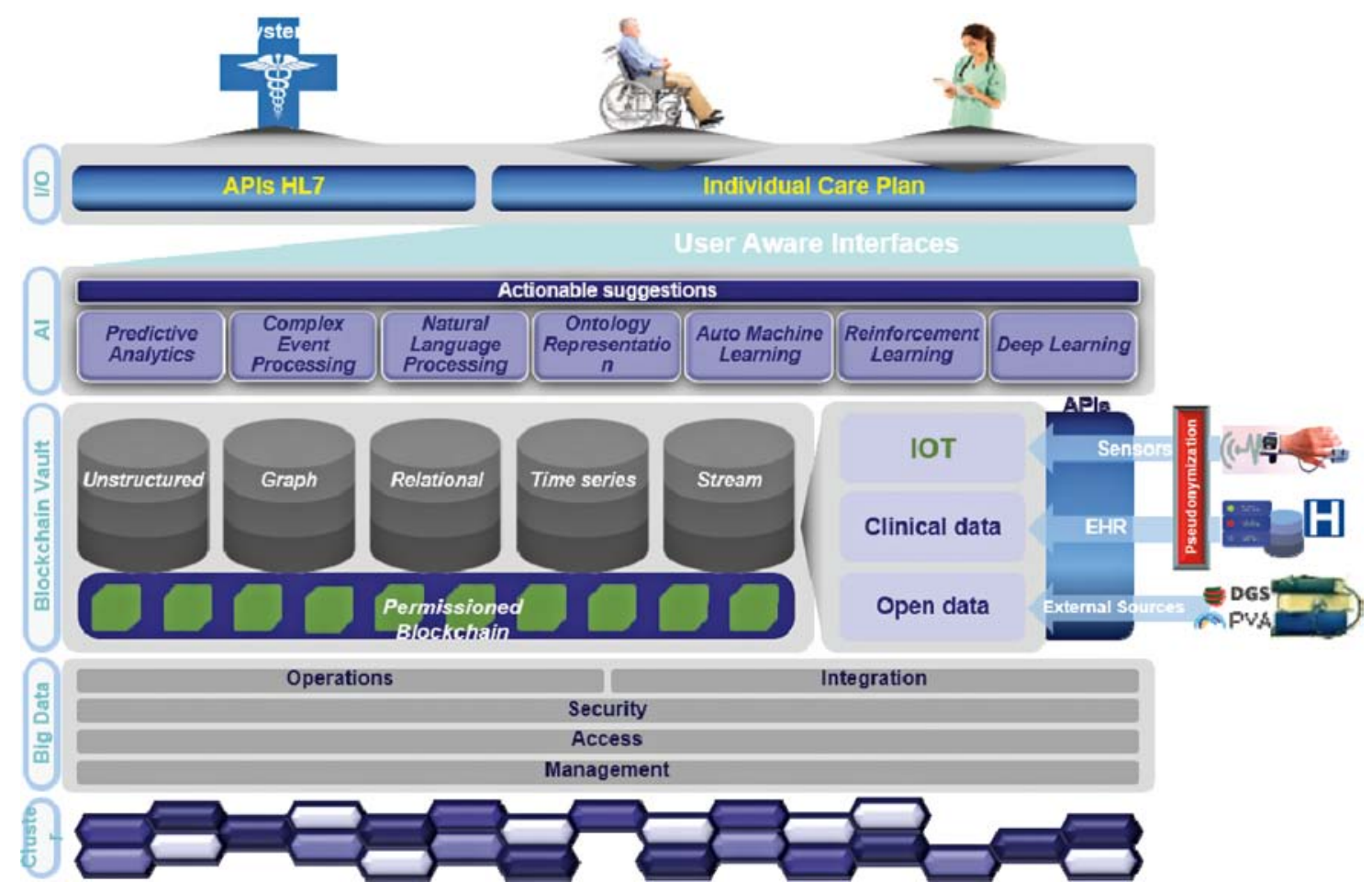

FIgURE 2. ALIE INFRASTRUCTURE

ed) Machine Learning processes that must be cleansed and directed for the most effective reasoning layer possible. An extreme example would be if a heart beating controller detects no beats it must, as quickly as possible, check if it is well positioned, battery in good condition, perform a double third party checking using an alternative life checker and if proven that the heart has possibly collapsed, activate the emergency services. This is all to be performed by the intelligence in the edge.

\section{IT structure in place}

To develop all the system for specific ALIEs in the currently globally most important multimorbidity conditions (OCPD, Diabetes and Coronary Chronic Disease), while maintaining the highest levels of information security and patient safety, we propose a multilayered system of systems depicted in the following diagram:

\section{Interactive session}

We will be demonstrating a conversational bot based in, an intelligent healthcare assistant, part of the User Aware Interfaces, that will activate a spoken dialogue in response of a possibly critical health event, a hypoglycemic alert, simulated in an ALIE where a diabetic person is monitored. ${ }^{3}$ We intend to show how the most recent technology of natural interfaces render a much more interesting way of interaction with the real world for increased healthcare providing value.

\section{RE FER E N C E S}

1. Vermesan O, Friess P. Building the Hyperconnected Society: Internet of things research and innovation value chains, ecosystems and markets. River Publishers; 2015. https://doi.org/ 10.13052/rp-9788793237988

2. "What Is Digital Twin Technology - And Why Is It So Important?" 06 Mar. 2017, https://www.forbes.com/sites/bernardmarr/2017/03/06/what-is-digital-twin-technology-and-whyis-it-so-important/.

3. Saias J, Quaresma P, Salgueiro P. (2012). Binli: An ontologybased natural language interface for multidimensional data analysis. Intelligent Information. 2012. http://search.proquest. com/openview/02d90b91dc3bf449471a2510b2407165/1?pqorigsite $=$ gscholar 


\section{Oral presentations}

\section{B5-01 \\ Interprofessional Educational Intervention in Dementia Care}

\author{
Alexander Kurz' ${ }^{1}$ Lea Pfaeffel ${ }^{1}$, Marina Boban ${ }^{2}$ \\ ${ }^{1}$ Technical University of Munich, School of Medicine, Germany \\ ${ }^{2}$ Referral Center for Cognitive Neurology and Neurophysiology, \\ University Hospital Centre Zagreb, School of Medicine, University of Zagreb, Croatia
}

In many countries of South Eastern Europe the quality of care for people with dementia and their families falls short of international standards. There is a lack of dementia-related knowledge and skills among health and social professionals, poor cooperation and coordination, as well as a shortage of important services such as day care centres or specialised nursing home units. Education of key occupational groups is considered to be key for improving dementia care and enhancing the quality of life of people with dementia and their informal carers. In 2017, dementia experts from ten South Eastern European countries teamed up with policy makers and businesses to create an educational intervention that simultaneously addresses multiple professions involved in dementia care. The intervention is unique in providing a common base of knowledge, a groundwork for understanding and collaboration, and a set of shared values in terms of a person-centred and holistic concept of dementia care. The intervention is in line with medical guidelines and complements existing or emerging national dementia strategies in the countries involved. It is delivered in a blended-learning format including traditional workshop materials and an e-learning programme. The development of the educational intervention and its evaluation in pilot actions in four countries is co-funded by the European INTERREG-Danube Transnational Programme from 2018 to 2021. The intervention is currently available in five languages (English, Bulgarian, Slovenian, Slovakian, Romanian). Dialogue with patient organisations and the policy level is currently ongoing in order to introduce the intervention in Croatia. We see the initiative's activities as a contribution to creating a "Better Future of Healthy Ageing".

\section{B5-O2}

\section{Commitment to Global Strategy and Action Plan on Ageing and Health}

\author{
Branko Kolarić', Tomislav Rukavina², Lovorka Bilajac², \\ Tanja Corić', Nada Tomasović-Mrčela ${ }^{1}$ \\ 'Department for Public Health Gerontology, Andrija Štampar Teaching Institute
of Public Health, Zagreb, Croatia
${ }^{2}$ School of Medicine, University of Rijeka, Croatia
}

Healthy Ageing is a concept that refers to developing and maintaining the functional ability that enables well-being in older age. It is a process that is implemented through the span of an entire life course. Functional ability is determined by the intrinsic capacity of an individual (i.e. the combination of all the individual's physical and mental capacities), the environment in which he or she lives (understood in the broadest sense and including physical, social and policy environments) and the interactions within them. Longer lives are one of our most remarkable collective achievements. By the year 2030, the number of people aged 60 years and older worldwide will be $34 \%$ higher, increasing from 1 billion in 2019 to 1.4 billion. The ageing of the population affects all aspects of society such as education, housing, health, long-term care, social protection, transport, information and communication, labour and financial markets as well as family structures and intergenerational ties. In this presentation, we will cover the strategic plans and actions regarding the implementation of Healthy Ageing, based on UN Political Declaration and Madrid International Plan of Action on Ageing, 2017 Lisbon Ministerial Declaration and WHO Global strategy and action plan on ageing and health. The five strategic objectives will be shortly presented: Commitment to action on Healthy Ageing in every country; Developing agefriendly environments; Aligning health systems to the needs of older populations; Developing sustainable and equitable systems for long-term care and Improving measurement, monitoring and research on Healthy Ageing. As a conclusion, and for the final challenge, we will also briefly tackle problems related to ageism and professional burn-out, in preparation for the new challenges and obstacles we will face in the future. This will enable us to be proactive and scale the ideologies of this concept. 


\section{B5-03}

\section{Oživi Me - from a Student Initiative to National Campaign}

Ivana Portolan Pajić, ${ }^{1,3}$, Filip Glavač ${ }^{2,3}$, Monika Tomas ${ }^{2,3}$

${ }^{1}$ City Office for Health, Zagreb, Croatia

2"StEPP" student association, School of Medicine, University of Zagreb, Croatia

3"Oživi me" National campaign, Croatia

Introduction. The Campaign "Oživi me" offered public educations on prompt bystander cardiopulmonary resuscitation held in every major Croatian city, with the goal of informing as many interested individuals as possible. Furthermore, informative workshops were held on a monthly basis at the Croatian Heart House Foundation with the goal of providing a deeper insight into the importance of prompt bystander cardiopulmonary resuscitation with the use of AED. Short description of practice change implemented. The Campaign has successfully raised the awareness of the importance of early recognition of the sudden cardiac arrest, and prompt bystander cardiopulmonary resuscitation. The Campaign has been accredited by several important institutions, such as the Ministry of Health, Croatian Institute for Public Health, as well as the University of Zagreb School of Medicine and the School of Dental Medicine. "Oživi me" ("Resuscitate me") has been supported by many public individuals, from celebrities to political and medical figures as well as other esteemed individuals. With its' influence, the Campaign raised a question of the neccessity of introducting the first aid and hands only CPR into the public school system curricula. Highlights.

The Campaign was announced and promoted via television and other electronic media by a promotional video and media interviews and presentations. An educational poster was created with the aim of placing it in public and gathering places. Through the interactive website any individual can educate him/herself through e-learning and instructive videos on handsonly cardiopulmonary resuscitation and adult basic life support with the use of AED and take part in an online educational quiz. Schools and other groups can take part in a Campaign-related mini-project "School with a heart" or "Dance to the Rhythm of the Heart" as well as "Life Hotspot". As a guidance method in case of an emergency situation, a mobile application was designed to help laymen assess the victim, call for help and deliver first aid. The application also consists of quick reminder of the standard first aid assessment, and resuscitation algorithm as well as an integrated AED map. Conclusions. Over 5000 laymen have been informed on early recognition and prompt first aid in case of sudden cardiac arrest. Over 70 AED devices were installed in various regions of Croatia according to the Campaign goals. Regarding to the latest feedback from the involved participants, 8 lives were saved as a result of "Oživi me" ("Reuscitate me") workshops and educations.

\section{Poster presentations}

No posters 